\title{
The Galactic Thick Disk: An Observational Perspective
}

\author{
Bacham E. Reddy \\ Indian Institute of Astrophysics, Bengaluru, 560034, \\ email: ereddy@iiap.res.in
}

\begin{abstract}
In this review, we present a brief description of observational efforts to understand the Galactic thick disk and its relation to the other Galactic components. This review primarily focused on elemental abundance patterns of the thick disk population to understand the process or processes that were responsible for its existence and evolution. Kinematic and chemical properties of disk stars establish that the thick disk is a distinct component in the Milky Way, and its chemical enrichment and star formation histories hold clues to the bigger picture of understanding the Galaxy formation.
\end{abstract}

Keywords. stars: FGK dwarfs, Stars: abundances, Stars: Kinematics, Galaxy: disk

\section{Introduction}

Deciphering the history of the birth and the growth of the Milky Way Galaxy is one of the major outstanding astrophysical problems. Detailed studies of our Galaxy would help to gain insights into the formation and evolution of other galaxies, and thereby large scale structure formation in the universe. As inhabitants, we have much better access to our Galaxy to resolve its building blocks: the stars. The properties of stars that constitute the Galaxy are clues to the processes involved in the making of the Milky Way Galaxy we see today. The observational data on stellar motions and photospheric abundances played decisive roles in the progression of our understanding of the Galaxy. The major, perhaps the first, quantitative study of stellar kinematic and chemical properties of a large sample of nearby dwarfs by Eggen, Lynden-Bell and Sandage (1962), known as ELS, laid the foundation for the Galaxy formation models. In this seminal paper ELS showed that the eccentricity of stellar orbits and the photometrically derived UV excess $(\delta(\mathrm{U}-\mathrm{B}))$, an indicator of metallicity, are correlated: stars with the largest excess (metalpoor) are found to be moving in highly elliptical orbits, where as the stars with little or no excess (solar metallicity stars) are found to have circular orbits. ELS attributed this to the rapid collapse of a proto-galactic cloud forming the halo quickly, and the rotationally supported disk component thereafter. Further studies, based on improved observations of globular clusters in the Galaxy, led to the fact that mergers play significant role in the evolution of the Galaxy (Searle \& Zinn 1978). Thanks to large scale surveys of both the kinematic and photometric properties of stars, we now know that the Galaxy consists of four major components: the halo, the thick disk, the thin disk and the bulge. There are excellent reviews which trace the progressive understanding of the subject (e.g; Gilmore 1989; Majewski 1993; Freeman \& Bland-Hawthorn 2002). In this review, we primarily concentrate on the thick disk component and its relation to the Milky Way galaxy. 


\section{The Galactic Thick Disk}

Evidence for a distinct component, the so called thick disk, in the disks first came from the data of external galaxies. Studies of brightness distribution as a function of distance from the galactic planes of some of the edge-on galaxies (NGC 4570, NGC 4350) required fitting of a third component, in addition to the usual two components (a thin disk and a bulge), for the fainter part (Burstein 1979), termed as the "thick disk" for its diffused or fluffy appearance. Shortly, thereafter a definitive evidence of the existence of the thick disk in the Milky Way Galaxy had emerged from the photometric data of a large sample of stars towards the South Galactic Pole (Gilmore \& Reid 1983). From the data of star counts or stellar density distribution as a function of distance from the Galactic plane, two distinct components were identified: one with a scale height of $300 \mathrm{pc}$ within $1 \mathrm{kpc}$ of the plane, and the second with a scale height of $1350 \mathrm{pc}$ which dominates the stellar density law in the Galaxy between $1 \mathrm{kpc}$ to about $5 \mathrm{kpc}$. The first one with a smaller scale height was identified as the old disk or the thin disk and the second component with a large scale height was identified as the thick disk analogous to the one found in the edge-on galaxies. The thick disk population is the same population of stars in the Galaxy that was known earlier, but not so well defined, as the intermediate population II or IPII (Strömgren 1964). Subsequent studies of photometric and metallicity data revealed that the thick disk population stars are metal-poor, old and show no evidence of metallicity gradients within the thick disk (e.g; Gilmore, Wyse \& Jones 1995). Thus, the concept of the thick disk is established and is termed as a major modification to the ELS model of rapid collapse for the formation of the Galaxy.

\section{Global properties}

Identification of Galactic stellar populations into distinct components relies, primarily, on five basic differences: location, structural properties, kinematic motions, age and chemical make-up. Until late 90s, studies mostly focused on defining the global properties of the thick disk like scale height, scale length, local normalization, metallicity distribution, kinematic properties and to some extent ages relative to the other components. Thanks to the Hipparcos space mission for accurate stellar astrometry, and the major ground based surveys like Radial Velocity Experiment (RAVE), Sloan Digital Sky Survey (SDSS), and 2 Micron Sky Survey (2MASS) it became abundantly clear that the thick disk exists and it has distinct global properties which are different from that of the thin disk, halo and the bulge. Since the halo and the bulge populations are known to differ both in morphology and kinematics from the disk populations, we concentrate on distinguishing two distinct populations within the Galactic disk: the thin disk and the thick disk.

Recently measured values of the thick disk population stars are summarized in Table 1. The thick disk has scale height in the range of $500 \mathrm{pc}$ to $1100 \mathrm{pc}$ which is two to four times of the thin disk $(300 \mathrm{pc})$, and its metallicity peaks at $[\mathrm{Fe} / \mathrm{H}] \approx-0.6$ dex compared to the thin disk which peaks at $[\mathrm{Fe} / \mathrm{H}] \approx-0.2$. Thick disk stars are older (8-12 Gyrs) compared to their counter parts in the thin disk stars $(<10$ Gyrs). Local normalization of the thick disk population relative to the thin disk population varies from survey to survey and which is in the range of $2 \%$ to $13 \%$. That is there are 2 to 13 thick disk stars for every 100 thin disk stars in the solar neighborhood. Volume limited (stars within the radius of 25 pc from Sun) survey by Fuhrman (2008) suggests even much larger proportion $(20 \%)$ of thick disk stars in the local neighborhood implying a massive thick disk. The population of the thick disk stars increases as one moves away from the Galactic plane 
Table 1. Summary of the Global and Kinematic properties of the Thick Disk

\begin{tabular}{lccc}
\hline Parameter & $\begin{array}{c}\text { Carollo et al. } \\
(2009)\end{array}$ & $\begin{array}{c}\text { Juric et al. } \\
(2008)\end{array}$ & $\begin{array}{c}\text { Siegel et al. } \\
(2002)\end{array}$ \\
\hline Scale height(kpc) & $0.51 \pm 0.04$ & $900 \mathrm{pc}$ & $940 \mathrm{pc}$ \\
Scale length (kpc) & $2.20 \pm 0.35$ & $3.6 \mathrm{kpc}$ & $\ldots$ \\
Local normalization & $15 \pm 7 \%$ & $\ldots$ & $8.5 \%$ \\
\hline & Carollo et al. & Soubiran et al. & Chiba \& Beers \\
\hline V ${ }_{\text {lag }}($ kms -1 & $38 \pm 2$ & $51 \pm 5$ & 20 \\
Velocity Ellipsoid & $(53 \pm 2,51 \pm 1,35 \pm 1)$ & $(63 \pm 6,39 \pm 4,39 \pm 4)$ & $(46 \pm 4,50 \pm 4,35 \pm 3)$ \\
Metallicity peak & -0.6 & -0.48 & \\
\hline
\end{tabular}

in the z-direction. The thick disk dominates other populations beyond $1.0 \mathrm{kpc}$. If the thick disk is a separate component one would also expect distinct kinematic motion $(U$, $V, W)$ for the thick disk population which is different from the other two components: the halo and the thin disk.

One of the potential discriminators of the Galactic components is their average rotational velocity $\left(\mathrm{V}_{\text {rot }}\right)$ or asymmetric drift velocity $\left(V_{\text {lag }}\right)$ with respect to the Galactic rotation of $220 \mathrm{~km} \mathrm{~s}^{-1}$. This has been known for a while (see Majewski 1993). As shown in Table 1, values of $V_{l a g}$ for the thick disk range anywhere between $20 \mathrm{~km} \mathrm{~s}^{-1}$ (Chiba \& Beers 2000) to $51 \mathrm{~km} \mathrm{~s}^{-1}$ (Soubiran et al. 2003). Large range in the measured $V_{\text {lag }}$ is, perhaps, due to the sample selection in the respective studies. However, $V_{\text {lag }}$ of $40-50$ $\mathrm{km} \mathrm{s}^{-1}$ is generally in good agreement with the other recent studies (e.g; Robin et al. 2003). Compare this with the halo population which has $\mathrm{V}_{\text {lag }} \approx 220 \mathrm{~km} \mathrm{~s}^{-1}$ (or statistically stationary w.r.t to the Galactic rotation) and with the thin disk of $\mathrm{V}_{\text {lag }} \approx 5-10 \mathrm{~km}$ $\mathrm{s}^{-1}$ (Robin et al. 2003). The measured velocity ellipsoid $\left(\sigma_{u}, \sigma_{v}, \sigma_{w}\right)$ of the thick disk is about twice that of the thin disk and half of the halo values. In all of the measurements, Gaussian distribution is assumed, and the non-Gaussian velocity distributions can't be ruled out.

In summary, as far as the Global properties of the thick disk are concerned, measurements based on different data sets and different methodologies agree with each other suggesting a level of overall confidence in characterizing the thick disk. Discontinuity in the luminosity function, metallicity distribution and the difference in rotational velocity are some key evidences for the distinct nature of the thick disk population in the disk of the Galaxy.

\section{Stellar Abundances of the Thick Disk}

Low mass main sequence dwarfs play an important role in tracing the sequence of events that took place during the Galaxy evolution. Unevolved dwarfs preserve the chemical composition of their natal clouds. Thus, the determination of photospheric abundances and their relative ratios is of great importance, in other words, chemical tagging of individual stars would help to understand the fundamental processes involved in the making of the Galaxy. In their review, Freeman \& Bland-Hawthorn (2002) stressed the importance of the differential abundance analysis for accurate abundance determinations. Sample selection is one of the important steps in this endeavour to keep the uncertainties at minimum. Dwarfs of spectral type $F$ and $G$ are well suited for accurate abundance determinations. In the stars of earlier spectral type, spectral lines are very broad due to higher rotational velocity, and some of them are either very weak or absent due to hotter temperatures. On the other hand, cooler stars have rich spectra with crowded regions 
Table 2. Summary of a few key abundances ratios for the kinematically selected stars the Halo, thick disk, MWTD, and thin disk. Data is adopted from a series of papers by Reddy et al. group.

\begin{tabular}{lcccc}
\hline$[\mathbf{X} / \mathbf{F e}]$ & Halo & MWTD & Thick Disk & Thin disk \\
\hline$[\mathrm{Mg} / \mathrm{Fe}]$ & $0.31 \pm 0.06$ & $0.32 \pm 0.07$ & $0.31 \pm 0.09$ & $0.09 \pm 0.05$ \\
{$[\mathrm{Si} / \mathrm{Fe}]$} & $0.23 \pm 0.06$ & $0.27 \pm 0.09$ & $0.23 \pm 0.15$ & $0.07 \pm 0.04$ \\
{$[\mathrm{Ni} / \mathrm{Fe}]$} & $0.02 \pm 0.04$ & $-0.08 \pm 0.09$ & $-0.08 \pm 0.11$ & $-0.02 \pm 0.02$ \\
{$[\mathrm{Eu} / \mathrm{Fe}]$} & $0.38 \pm 0.13$ & $0.44 \pm 0.24$ & $0.35 \pm 0.15$ & $0.12 \pm 0.10$ \\
\hline
\end{tabular}

making it difficult to analyze, and hence larger uncertainties. Another important consideration is the selection of elements. The $\alpha$-elements like $\mathrm{O}, \mathrm{Mg}, \mathrm{Si}, \mathrm{Ca}$ or $\mathrm{Ti}$ are thought to be predominantly produced in the short lived massive star explosions (SNII) and elements like Fe, Ni etc. are the main products from the long lived low mass star explosions (SNIa). SNII are more frequent in the early epochs and SNIa starts contributing much later. Thus, ratio of $[\alpha / \mathrm{Fe}]$ is a tracer of the chemical evolution and star formation rates in the Galaxy as well as a indicator of relative frequency of SNII to SNIa explosions.

The abundance study of the Galactic disk stars of Edvardsson et al. (1993) is the first major work in this direction. They have analyzed high resolution spectra of $189 \mathrm{~F}$ - and Gdwarfs and measured abundances of 12 elements, which provided important constraints for the Galactic chemical evolutionary models. They have noticed significant scatter in $[\alpha / \mathrm{Fe}]$ versus $[\mathrm{Fe} / \mathrm{H}]$, particularly, below $[\mathrm{Fe} / \mathrm{H}] \approx-0.4$. Stars with relatively higher $[\alpha / \mathrm{Fe}]$ ratios found to be preferentially more eccentric and closer to the Galactic center. This was attributed to an efficient star formation rate in the inner disk. Surprisingly, the possibility of the thick disk population to the observed scatter in the abundances was never raised in the entire lengthy paper. By the late 1990s, it became increasingly clear that the thick disk harbours a stellar population whose chemical history is distinct from the rest of the components in the Galaxy, particularly the thin disk. Fuhrman (1998) study of carefully selected and unbiased, but volume limited, sample of $\mathrm{F}$ and $\mathrm{G}$ dwarfs indicated that stars with intermediate kinematic properties show higher $[\mathrm{Mg} / \mathrm{Fe}]$ ratio compared to the thin disk stars in the overlapping metallicities. This was confirmed from the detailed study of many more elements but for a small kinematically pre-selected sample of 10 stars (Prochaska et al. 2000). Abundance ratios of $[\alpha / \mathrm{Fe}]$ found to be higher compared to the thin disk stars but very similar to halo and bulge stars. Contrary to the above results, Chen et al. (2000) study of pre-selected samples of thick disk showed continuous chemical evolution from the thick to the thin disk populations.

\section{Evidence for the distinct chemical history}

Is the thick disk a discrete entity in the Galaxy? Does it evolve from the thin disk to the thick disk or vice versa? What is the metallicity range of the thick disk? Answers to these questions will help to piece together the puzzle of the thick disk and its relation to the rest of the Galaxy. As noted in the above section, there seems to be evidence that the chemical history of the thick disk is different, at least from that of the thin disk. But this needs to be validated from the larger samples of stars and more number of elements of different nucleosynthetic history. Also, to put the thick disk in the context of the Galaxy evolution, uniform study of samples of stars from other components is essential. Precisely, this was pursued independently, in the recent studies (Reddy et al. 2003, Reddy, Lambert, Allende Prieto 2006, Reddy \& Lambert 2008; Bensby et al. 2003, 2005, 2007; Fuhrman 1998, 2008). Bulk of the information on the abundances of the thick disk and thin disk populations is drawn from the above studies particularly from the two 
groups: Reddy et al. and Bensby et al. These studies are complimentary to each other as the sample stars of Bensby et al. are drawn from the southern hemispheres whereas the samples in the studies of Reddy et al. are from the northern hemisphere. Common stars among the two studies are very few. However, the agreement between the two results,in most cases, is extremely good indicating the maturity of the different model atmospheres and the analysis techniques.

In the studies of Reddy et al. $(2003,2006)$ a carefully pre-selected sample, based on kinematic properties, of about 400 nearby dwarfs belong to the thin disk, the thick disk, or the halo were subjected to high resolution spectroscopy. Stars were grouped into the thin disk, thick disk and the halo populations based on their kinematic definitions and relative fraction of each of these populations in the solar neighbourhood. Quantitative abundances of 22 to 26 elements were determined with uncertainties as low as 0.04 0.06 dex for most of the key elements. The mean $[\mathrm{X} / \mathrm{Fe}]$ ratios are given in Table 2 for the thick disk, thin disk and the halo stars. Results for a few key elements are shown in Figure 1. Similar studies based on similar number of stars were carried out in a series of papers by Bensby et al. group. In general, results from both the studies are in good agreement. Some of the Key conclusions from these results are: a) Abundance ratios of elements that are known to be the dominant yields from SNII are clearly larger than their counter parts in the thin disk in the overlapping metallicity $(-0.3--0.8$ dex $)$ implying a distinct chemical history for the thick disk from that of the thin disk. However, the

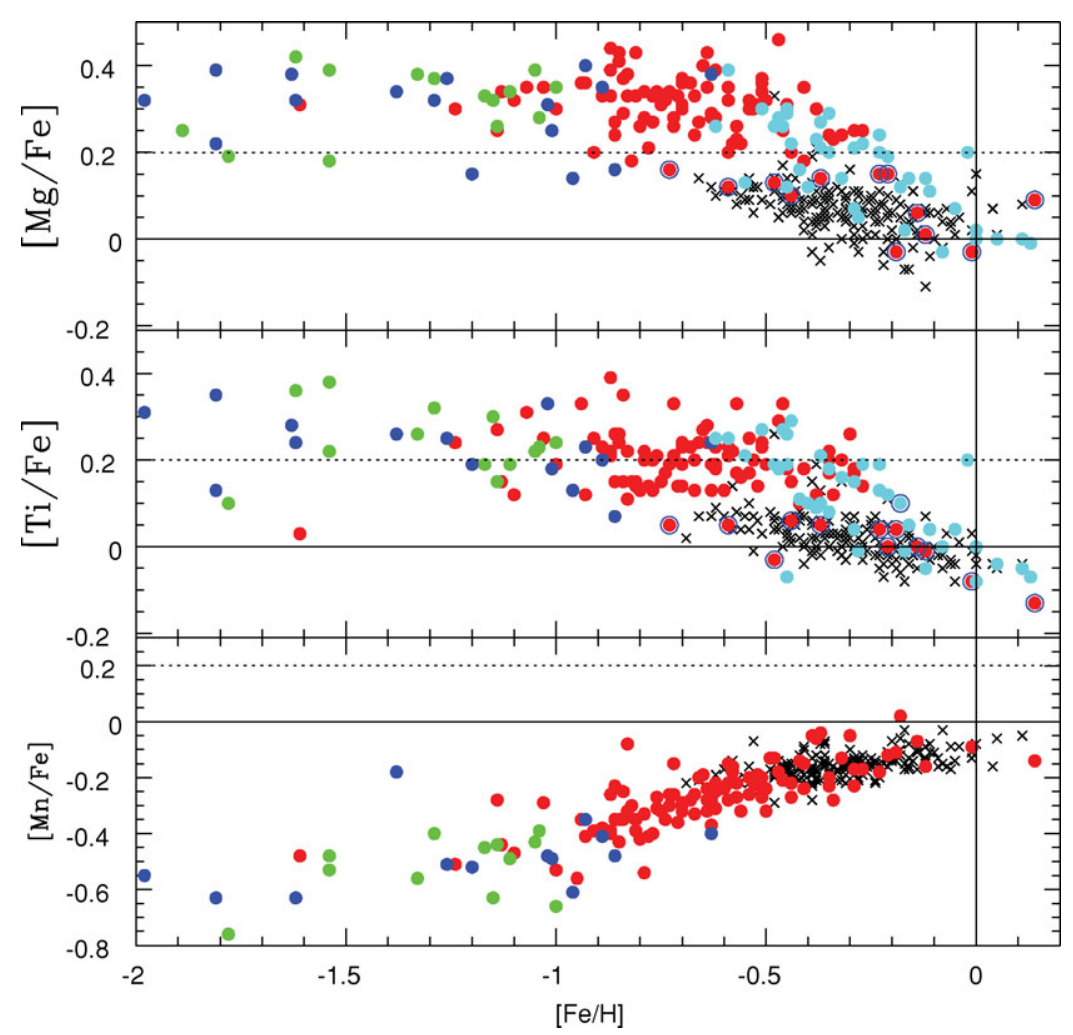

Figure 1. Abundances ratios $[\mathrm{Mg} / \mathrm{Fe}],[\mathrm{Ti} / \mathrm{Fe}]$ and $[\mathrm{Mn} / \mathrm{Fe}]$ versus $[\mathrm{Fe} / \mathrm{H}]$ for samples of the thick disk (red circles), the thin disk (black crosses) and the halo stars (blue circles). Light blue and green circles are shown for stars of metal-weak and metal-rich thick disks, respetively. TKTA stars are shown as red circles embedded in blue circles. 
mean abundance ratios of elements of thick disk stars are indistinguishable from those of MWTD or halo stars and even the bulge stars ( e.g; Meléndez et al. 2008). The similarity of the thick disk abundance ratios with that of the halo and bulge may suggest that the three components are similarly old and had a rapid star formation. b) The run of $[\alpha / \mathrm{Fe}]$ with $[\mathrm{Fe} / \mathrm{H}]$ for stars of the thick disk shows little or no slope against $[\mathrm{Fe} / \mathrm{H}]$ suggesting rapid formation of the thick disk. Slight slope towards higher metallicities is attributed to $\alpha$-element yield dependence on the initial metallicities of SNII. c) Another important outcome of the analysis is that the star-to-star scatter in the trends which is comparable to the scatter expected from the measurements indicating stars both in the thin as well as in the thick disk formed from the well mixed gas, and d) Age estimates indicate that the stars of the thick disk are older on average (8-12 Gyrs) compared to the thin disk stars $(\mathrm{t}<10$ Gyrs). Age results show that 2-3 Gyrs elapsed between the first stars of the thin disk and the first stars of the thick disk suggesting sufficient time for the SNIa role in the chemical history of the thick disk.

\section{Metal-Rich end of the thick disk}

Is thick disk evolving similar to the thin disk or is it a frozen entity in the Galactic disk? Evidence of delayed SNIa contribution in the thick disk was first proposed by Bensby et al. (2003). The claim of the so called "Knee" connecting thick disk stars from high $[\alpha / \mathrm{Fe}]$ trend below $[\mathrm{Fe} / \mathrm{H}]=-0.3$ to thick disk stars of solar $[\alpha / \mathrm{Fe}]$ values above $[\mathrm{Fe} / \mathrm{H}]$ $=-0.2$ was disputed first by Reddy et al. (2006) and later by Ramírez et al. (2007). Confirmation of presence or absence of the "knee" in the $[\alpha / \mathrm{Fe}]$ trend with $[\mathrm{Fe} / \mathrm{H}]$, and its smooth merging with the thin disk trend is of significance. Such an evidence will constrain the thick disk chemical enrichment models.

Evidence of SNIa contribution to the chemical history of the thick disk was not very clear in the Reddy et al. (2006) abundance survey of the thick disk. This was partly due to the presence of a different population with thick disk kinematics but with thin disk chemistry (Reddy et al. 2006) and partly due to lack of sufficient number of stars to form the "knee". As shown in Fig 1 (red symbols: Reddy et al. 2006), one could see a hint of drop in the $[\alpha$-elements $/ \mathrm{Fe}]$ trends at $[\mathrm{Fe} / \mathrm{H}]=-0.35$ and a few thick disk stars above $[\mathrm{Fe} / \mathrm{H}] \approx-0.2$ with very similar abundances of thin disk. Evidence for the drop in the $[\alpha / \mathrm{Fe}]$ was overlooked to explain the thick disk stars below $[\mathrm{Fe} / \mathrm{H}]=-0.35$ with thin disk abundances. Together they were termed as TKTA stars (Fig 1: red symbols embedded in blue circles) for thick disk kinematics and thin disk abundances. Ramírez et al. (2007) explored the thick disk chemical evolution using $\mathrm{O}$ abundance of about 500 dwarfs. From the results of detailed LTE and NLTE analysis they have suggested that the thick disk chemical evolution ends at $[\mathrm{Fe} / \mathrm{H}] \approx-0.3 \mathrm{dex}$, and the proposed existence of a knee in the $\mathrm{O}$ abundance trend was challenged. This required further studies to settle the issue.

Bensby et al. (2007) undertook a systematic study of thick disk stars in the metallicity range of $-1 \leqslant[\mathrm{Fe} / \mathrm{H}] \leqslant+0.4$ with sufficient number of thick disk stars above $[\mathrm{Fe} / \mathrm{H}]=$ -0.35 . Results showed strong evidence that the thick disk extends at least to the solar metallicities and the drop in the $[\mathrm{O} / \mathrm{Fe}]$ pattern occurred at $[\mathrm{Fe} / \mathrm{H}=-0.35$. This was also confirmed from the new sample based on several elements (Reddy et al. 2009 and Bensby et al. in this volume). In Figure 1, our new kinematically chosen thick disk sample stars are shown in light blue. They connect thick disk stars (red circles) of high $[\alpha / \mathrm{Fe}]$ trend with thin disk stars of solar $[\alpha / \mathrm{Fe}]$ trend. Results in Fig 1 and Fig 2 highlight the complexity of the disk structure: the TKTA population persists in the new analysis, confirming the earlier claim by Reddy et al. (2006). The results in Fig 2 also suggest that 
there seems to be multiple channels that are connecting thick disk trends with that of the thin disk.

\section{Metal-Weak end of the thick disk}

There are not many studies that focused on the Metal Weak Thick Disk stars (MWTD). Morrison et al. (1990) were the first to point out stars that are very similar to the disk kinematics but significantly metal-poor, $[\mathrm{Fe} / \mathrm{H}]<-1.0$. Later, this turned out to be not real. The recalibration of the metallicities suggested that the so called metal weak thick disk stars were in fact found to have metallicities $[\mathrm{Fe} / \mathrm{H}]>-1.0$ (Ryan \& Lambert 1995; Twarog \& Anthony-Twarog 1996). Stronger evidence for the MWTD appeared in the analysis of large data sets of metal-poor stars (Chiba \& Beers 2000). The $[\mathrm{M} / \mathrm{H}]$ versus rotational velocity relations suggested stars as metal-poor as $[\mathrm{M} / \mathrm{H}]=-1.7$ have disk like velocities of $V_{l a g}>70 \mathrm{~km} \mathrm{~s}^{-1}$ slightly higher than the values estimated for the canonical thick disk and much lower than the halo population $\left(V_{l a g} \approx 220 \mathrm{~km} \mathrm{~s}^{-1}\right.$ ) (see Table 1$)$.

Isolation of the MWTD and determination of its status either as a metal-poor tail of the thick disk, or a collection of halo stars or a discrete Galactic component is important and worth to pursue to gain insights into the early epochs of the disk formation. Establishing the MWTD thick disk as a discrete entity would provide another evidence for the hierarchical galaxy formation. On the other hand, establishing it as a metal-poor tail of the thick disk would tell us the star formation history of the thick disk. An attempt was made to systematically search for MWTD and study their chemical abundance pattern in relation to the thin disk, thick disk and halo populations (Reddy \& Lambert 2008). For this study they made use of the two catalogues (Arifyanto et al. 2005; Schuster et al. 2006) to search for MWTD. From the combined catalogue of about 1700 stars, they have chosen the criteria for the MWTD: a) $[\mathrm{M} / \mathrm{H}]<-1.0$, (b) rotational velocity $\mathrm{V}_{r}>100 \mathrm{~km}$ $\mathrm{s}^{-1}$ (or $V_{\text {lag }}>120 \mathrm{~km} \mathrm{~s}^{-1}$ ), (c) $\left|W_{L S R}\right| \leqslant 100 \mathrm{~km} \mathrm{~s}^{-1}$ and $\left|U_{L S R}\right| \leqslant 140 \mathrm{~km} \mathrm{~s}^{-1}$. First condition eliminates contamination of stars from the thin disk and conditions $\mathrm{b}$ and $\mathrm{c}$ significantly enhance the proportion of MWTD to the halo stars.

High resolution abundance analysis study showed no clear difference in any elemental abundance trends, among several elements examined, between stars of the MWTD, halo or the canonical thick disk (see Table 2). This raises three possibilities; a) MWTD is a metal-poor end of the thick disk and shows no distinguishable chemical abundance difference as the thick disk formed more rapidly, b) MWTD is a discrete entity with indistinguishable abundances either from the thick disk or halo populations. The difference in age between the three populations is too small ( 1-2 Gyrs) to reflect differences in their chemical composition, and c) finally MWTD stars are basically a collection of halo stars with disk like kinematics. None of these possibilities could be ruled out at present as far as the MWTD is concerned. To establish the MWTD status, one may require large data sets with reliable kinematics from accurately measured distances, proper motions and radial velocities. Some of these efforts are underway. In a recent study Carollo et al. (2009) explored the full space motions for a sample of about 17000 stars in a local volume within $4 \mathrm{kpc}$ of the Sun. Photometric, low resolution spectroscopic, and proper motion data were taken from SDSS. In the metallicity regime of $-1.7<[\mathrm{Fe} / \mathrm{H}]<-1.0$ and in the interval of vertical distance from the Galactic plane $1<|Z|<2 \mathrm{kpc}$, observational data required to account for two distinct populations: inner halo $\left(\mathrm{V}_{\text {rot }} \approx 0\right)$ and MWTD with $\mathrm{V}_{\text {rot }} \approx$ $100-150 \mathrm{~km} \mathrm{~s}^{-1}$ ( or $\mathrm{V}_{\text {lag }} \approx 120-70 \mathrm{~km} \mathrm{~s}^{-1}$ ). They suggest that the MWTD is a separate component and is different from the canonical thick disk. This is a step forward to resolve the issue. However, this is based on a single paramete i.e., rotational velocity lag between the two thick disk stars. The presence of a large kinematic and spacial overlapping with 
the halo and the canonical thick disk, and the expected large uncertainties in derived astrometry will require additional studies to fully resolve the status of MWTD. Accurate astrometry for large samples and followed by accurate abundance study would certainly help to unravel the origin thick disk over its full metallicity range.

\section{Thick disk in the context of the Galaxy formation}

In the last two decades, a wealth of observational information was collected not only on the thick disk of the Milky Way Galaxy but also on the thick disks of many edge-on galaxies (e.g; Yochin \& Dalcanton 2008). Observational results deduced from various data sets by different groups are consistent, and they put stringent constraints on the theoretical modeling of the thick disk origin. To put the thick disk in the overall perspective of the Milky Way Galaxy formation, theoretical modelling has to take into account all the well established observational parameters: a) scale heights, b) local relative density of the thick disk, d) significantly older (8-12 Gyrs) age of thick disk stars compared to the thin disk stars of similar metallicity, d) distinct kinematic properties, e) no noticeable velocity as well as metallicity gradients as a function of vertical distance or age, f) distinct metallicity distribution: thick disk peaks at $-0.6 \mathrm{dex}$, thin disk at -0.2 dex and the halo at -1.6 dex, g) distinctly higher $[\alpha / \mathrm{Fe}]$ and $[\mathrm{Eu} / \mathrm{Fe}]$ ratios from that of the thin disk, $\mathrm{h}$ ) little or no gradient in the $[\alpha / \mathrm{Fe}]$ over a large range of metallicity, $-1.2<[\mathrm{Fe} / \mathrm{H}]<-0.3$, i) drop in the $[\alpha / \mathrm{Fe}]$ ratios at $[\mathrm{Fe} / \mathrm{H}] \approx-0.35$ dex and smooth merging with the thin disk ratios at about solar metallicities, $\mathrm{j}$ ) very little/no cosmic scatter in the abundance ratios over the large $[\mathrm{Fe} / \mathrm{H}]$ range, $\mathrm{k}$ ) existence of kinematically thick disk stars with thin disk abundance trends over the full metallicity range of the thin disk. Unfortunately, the status of the metal-poor end of the thick disk is yet to be understood,otherwise, it would have been an important constraint.

Ever since the existence of thick disk was established in disk galaxies as well as in the Milky Way, variety of theoretical models were proposed to explain the process that was responsible for the thick disk. Broadly, one could group these into two categories: top down and bottom up models. Briefly, in the top down models, thick disk is formed either through a rapid or dissipative collapse of protogalactic clouds. In this scenario halo

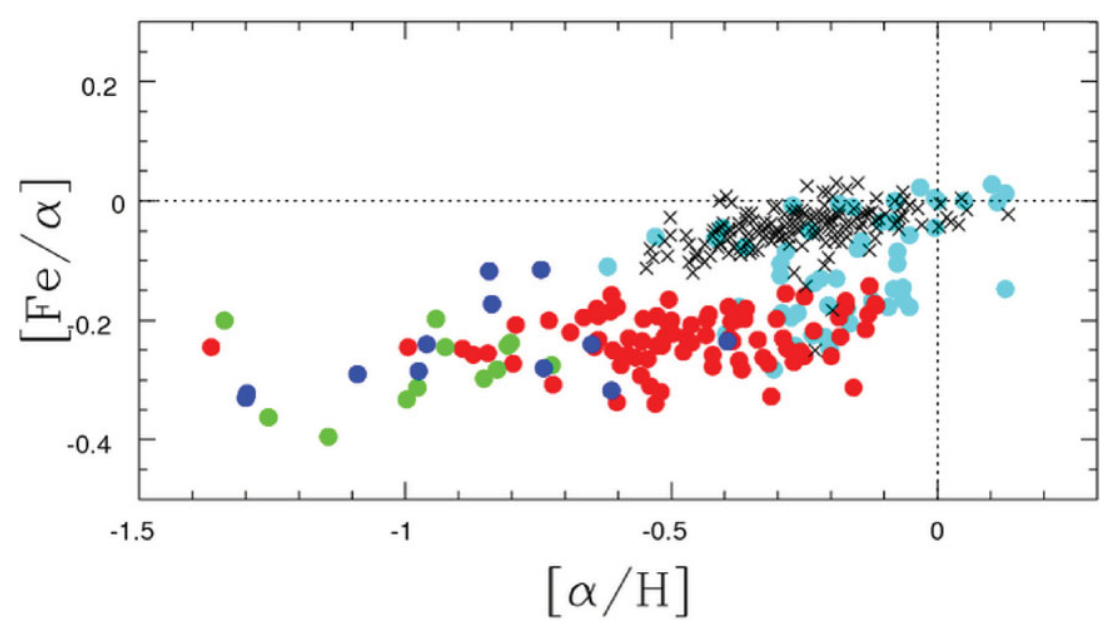

Figure 2. Abundances ratio $[\mathrm{Fe} / \alpha]$ versus $[\alpha / \mathrm{H}]$ for samples of the thick disk, the thin disk and the halo stars. Colour and symbol coding are same as those for in the Figure 1. 
forms first, then the thick disk, and then the thin disk. Top down models, by their nature, predict gradients both in abundances and kinematics, and do not predict distinct chemical abundances of the thick disk from the thin disk over the overlapping metallicities. Top down models fail to meet many of the well defined observational parameters listed above. For an exhaustive list of different models and their full description, readers are advised to refer to Majewski (1993) and references therein.

In the case of bottom up models thin disk already exists, and the thick disk forms later either through satellite mergers, accretion of debris or through radial mixing. These models are successful in predicting many of the observed properties of the thick disk. It may not be exaggerating to say that all the three possibilities may have contributed to the formation of the thick disk. Simulations of galaxy formation through hierarchical build up based on the $\Lambda$ CDM universe are regularly reported in the literature. Many of these simulations predict emergence of thick disk in the galactic disk, and some of its distinct properties from the thin disk and the halo. Quinn, Hernquist \& Fullagar (1993) proposed that satellite mergers heat the preexisting thin disk but not the gas. Thick disk stars are primarily the fossils of the thin disk. These models predict some of the distinct global properties like scale height and kinematic properties of thick disk stars. Later thin disk forms from the reformed gas from the merged satellites. Models predict only mild reduction in the rotational velocity, and do not account for the chemical enrichment of the thick disk from the delayed SNIa. Thin disk heating by mergers was probed further ( Kazantzidis et al. 2008) by including massive satellites to heat the thin disk sufficiently to form the thick disk. Massive satellites were more frequent at redshifts above $\mathrm{z} \approx 1$ which corresponds to the age of the thick disk in the $\Lambda$ CDM. Alternatively, Abadi et al. (2003) simulations of Galaxy formation in $\Lambda$ CDM universe, resulted a thick disk consisting mostly of stars from the satellite debris and a few through accretion. A clear distinction between dynamically cold disk of stars on nearly circular orbits and a thicker disk with orbital parameters in between the thin disk and the halo emerged. Results help to explain the existence of non-negligible fraction of thin disk stars which pre-date the last major merger history. However, the chemical evolution of the thick disk in the simulations in this framework need to be probed.

In a series of papers Brook et al. (2007 and references therein) proposed the formation of thick disk through heating of the thin disk by gas rich mergers and starburst during the merger process. Starburst during the merger accounted the observed thick disk properties like kinematic dispersions, higher $[\alpha / \mathrm{Fe}]$, and rapid star formation. Rapid star formation ensures higher $[\alpha / \mathrm{Fe}]$ even at higher metallicities with significantly much older age compared to thin disk stars with similar metallicities but much younger. The models also predict very low velocities for the merged stars and some of them have counter rotation. Though, simulations predict trend of decreasing of $[\alpha / \mathrm{Fe}]$ with $[\mathrm{Fe} / \mathrm{H}]$ but stops at much higher $[\alpha / \mathrm{Fe}] \approx 0.2$ dex even at solar metallicity. Simulations do not predict observed drop in $[\alpha / \mathrm{Fe}]$ which is the signature of SNIa to the thick disk chemical enrichment (see Figures $1 \& 2)$.

Radically different from the merger/accretion scenarios, recent studies focused on creating the thick disk out of the Galactic disk without accretion from outside. Radial migration of stars from inner to outer radii and the scattering of stars by spiral structure and the molecular clouds could explain many of the observed morphological, kinematical and chemical properties of the thick disk (e.g; Haywood 2008, Schonrich \& Binney 2009). Though, this is an interesting exercise, there are many assumptions like rate of infall and its distribution, and scattering time scales that need to be constrained further. Models 
based on radial mixing may find it difficult to explain the evidence of counter rotating thick disk stars that are seen in some of the spiral galaxies.

\section{Conclusions}

Significant progress has been made, over the last two decades, towards establishing various observational properties. However, we still lack information on the metal-poor side of the thick disk. This has implications to favour a particular merging model. The issue of understanding the MWTD is related to accurate astrometry for a large number of stars at relatively farther distances. Hopefully, this would be resolved with the proposed GAIA space mission data expected to be available in 2015/16. Systematic studies of thick disk in large number of spiral galaxies with varying mass would help to constrain the mass of the merging satellites in the simulations of a particular galaxy with a particular thick disk to thin disk mass ratio. As far as decoding the disk components in other galaxies, we require more powerful tools like next generation large aperture telescopes which are at the horizon. As shown in Figure 2, thick disk structure is much more complex and observations point to multiple process for this structure: mergers, accretion of stars from the debris, heating of the thin disk through scattering, and radial mixing. Hopefully, in the near future, an unified model will be developed that would match all the well defined observed parameters.

\section{Acknowledgements}

I thank SOC for inviting and giving me this opportunity to review the thick disk and LOC for their superb arrangements at the meeting. I thank INSA, IAU and IIA for providing the funds to attend the meeting.

\section{References}

Abadi, M. G., Navarro, J., Steinmetz, M., \& Eke, V. R. 2003, ApJ, 597, 21

Arifyanto, M. I., Fuchs, B., Jahrei, H., \& Wielen, R. 2005, AA, 433, 911

Bensby, T., Feltzing, S., \& Lundström, I. 2003, $A \& A$, 410, 527

Bensby, T., Feltzing, S., Lundström, I., \& Ilyin, I. 2005, A\&A, 433, 185

Bensby, T., Zenn, A. R., Oey, M. S., \& Feltzing, S. 2007, ApJl, 663, 13

Brook, C., Richard, S., Kawata, D., Martel, H., \& Gibson, B. K. 2007, ApJ, 658, 60

Burstein, D. 1979, ApJ, 234, 829

Carollo et al. 2009, arXiv, 0909.3019

Chen, Y. Q., Nissen, P. E., Zhao, G., Zhang, H. W., \& Benoni, T. 2000, AEAAS, 141, 491

Chiba, M. \& Beers, T. C. 2000, AJ, 119, 2843

Edvardsson, B., Andersen, J., Gustafsson, B., Lambert, D.L., Nissen, P. E., \& Tomkin, J. 1993, $A \& A, 275,101$

Eggen, O. J., Lynden-Bell, D., \& Sandage, A. R. 1962, ApJ, 136, 748

Freeman, K. \& Bland-Hawthorn, J. 2002, ARAA, 40, 487

Fuhrmann, K. 1998, A\&A, 338, 161

Fuhrmann, K. 2008, MNRAS, 384, 173

Gilmore, G. \& Reid, N. 1983, MNRAS, 202, 1025

Gilmore, G., Wyse, R. F. G., \& Kuijen, K. 1989, ARAA, 27, 555

Gilmore, G., Wyse, R. F. G., \& Jones, J. B. 1995, AJ, 109, 1095

Haywood, M. 2008, MNRAS, 388, 1175

Jurić et al. 2008, ApJ, 673, 864

Kazantizidis, S., Bullock, J. S., Zenter, A. R., Kractsov, A. V., \& Mosutakas, L. A. 2008, ApJ, 688,254

Majewski, S. R. 1993, ARAA, 31, 575 
Meléndez et al. 2008, A\& $A, 484, \mathrm{~L} 21$

Morrison, H. L., Flynn, C., \& Freeman, K. C. 1990, AJ, 100, 1191

Prochaska, J. X., Naumov, S. O., Carney, B. W., McWilliam, A., \& Wolfe, A. M. 2000, AJ, 121,2513

Quinn, P. J., Hernquist, L., \& Fullagar, D. P. 1993, ApJ, 403, 74

Ramírez, I., Allende Prieto, C., \& Lambert, D. L. 2007, A\& A, 465, 271

Reddy, B. E., Tomkin, J., Lambert, D. L., \& Allende Prieto, C. 2003, MNRAS, 340, 304

Reddy, B. E., Lambert, D. L., \& Allende Prieto, C. 2006, MNRAS, 367, 1239

Reddy, B. E. \& Lambert, D. L. 2008, MNRAS, 364, 25

Robin, A. C., Reylé, C., Derriére, S., \& Picaud, S. 2003, A\&A, 409, 523

Ryan, S. G. \& Lambert, D. L. 1995, AJ, 109, 2068

Schönrich, R. \& Binney, J. 2009, MNRAS, 396, 203

Schuster, W. J., Moiyinho, A., Mrquez, A., Parrao, L., \& Covarrubias, E. 2006, A\&A, 445, 939

Searle, L. \& Zinn, R. 1978, ApJ., 225, 357

Siegel, M. H., Majewski, S. R., Reid, I. N., \& Thompson, I.B 2002, ApJ., 578, 151

Soubiran, C., Bienayme., \& Siebert, A. 2003 A\&A, 398, 141

Strögren, B. 1964 Astrophys. Norvegica, 9, 333

Tawrog, B. A. \& Anthony-Twarog, B.J 1996, AJ, 111, 220

Yoachim, P. \& Dalcanton, J. 2009, ApJ, 682, 1004 